\title{
Diagnostic stability was higher in schizophrenia than other psychoses
}

Schwartz JE, Fennig S, Tanenberg-Karant M, et al. Congruence of diagnoses 2 years after a first-admission diagnosis of psychosis. Arch Gen Psychiatry 2000 Jun;57:593-600.

\section{QUESTION: In patients with a first admission diagnosis of psychosis, how stable is the diagnosis?}

\section{Design}

An inception cohort of patients with follow up of 6 and 24 months (Suffolk County Mental Health Project).

\section{Setting}

12 psychiatric inpatient facilities in Suffolk County, Long Island, New York, USA.

\section{Patients}

547 patients (median age 28 y, 57\% men, 86\% non-African-American) who were $15-60$ years and had a first hospital admission for psychosis (DSM-IV criteria). Exclusion criteria were first psychiatric hospital admission $>6$ months before current admission, moderate or severe mental retardation, or inability to speak English.

\section{Assessment of prognostic factors}

Psychosis diagnoses were bipolar disorder (BPD) and major depressive disorder (MDD) with psychotic features, schizophrenia spectrum disorders (SSD), and other non-affective psychoses based on DSM-IV criteria. Data on demographic characteristics, family history of psychiatric illness, clinical history, and initial hospital admission were assessed.

\section{Main outcome measures}

Psychosis diagnoses at 6 and 24 months and risk factors associated with a change to SSD between 6 and 24 months.

\section{Main results}

More patients diagnosed with SSD at 6 months had an unchanged diagnosis at 24 months than did patients with other diagnoses $(90 \%$ for SSD $v 83 \%$ for BPD, $\{\mathrm{p}=0.04\}^{*} ; 74 \%$ for MDD, $\{\mathrm{p}<0.001\}^{*}$; and $63 \%$ for other psychoses, $\left.\{\mathrm{p}<0.001\}^{*}\right)$ (table). Risk factors associated with a change to SSD diagnosis were facility diagnosis of schizophrenia, negative symptoms, longer time between psychosis onset and initial hospital admission, antipsychotic medication at discharge, longer duration of initial hospital stay, poorer adolescent social adjustment, and lifetime substance use disorder.

\section{Conclusion}

In patients with a first admission diagnosis of psychosis, more patients with schizophrenia spectrum disorders, in particular schizophrenia, had a stable diagnosis than did those with bipolar disorder, major depressive disorder, or other non-affective psychoses.

*p Values calculated from data in article.
Source of funding: in part, US National Institute of Mental Health.

For correspondence: Dr J Schwartz, Department of Psychiatry, Room 139, Putnam Hall-South Campus, SUNY-Stony Brook, Stomy Brook, NY 11794-8790, USA. Fax $+16316329433$.

Stability of psychosis diagnosis between 6 and 24 months

\begin{tabular}{llll} 
Diagnosis & $\begin{array}{l}6 \text { month } \\
\text { assessment }\end{array}$ & $\begin{array}{l}24 \text { month } \\
\text { assessment }\end{array}$ & $\begin{array}{l}\text { Percentage with } \\
\text { unchanged diagnosis }\end{array}$ \\
$\begin{array}{clll}\text { Schizophrenia spectrum } \\
\text { disorders (SSD) }\end{array}$ & 189 & $\begin{array}{l}216-(171 \text { same) } \\
12 \text { from BPD } \\
9 \text { from MDD } \\
24 \text { from other }\end{array}$ & $90 \%$ \\
& & $138-(117$ same) & $83 \%$ \\
\hline Bipolar disorder (BPD) & 141 & 5 from SSD & \\
& & 7 from MDD & \\
& & 9 from other & \\
\hline Major depressive disorder & 103 & $93-(76$ same $)$ & $74 \%$ \\
(MDD) & & 7 from SSD & \\
& & 9 from BPD & \\
& & $100-(72$ same $)$ & $63 \%$ \\
\hline Other non-affective & 114 & 6 from SSD \\
psychoses & & 11 from BPD & \\
& & 11 from MDD & \\
& &
\end{tabular}

\section{COMMENTARY}

In the absence of biological correlates, stability of diagnoses over time currently serves as the best diagnostic standard to validate psychiatric diagnoses. The study by Schwartz et al confirms the results of studies with longer follow up periods that show greater diagnostic stability if operationalised criteria are used for the diagnosis of schizophrenia. ${ }^{1}$ The results also emphasise the need for clinicians and researchers to adopt a longitudinal, as opposed to a cross sectional, perspective and to use multiple sources of information to avoid misdiagnoses. Changes in diagnoses over time are then more likely to be caused by the evolution of the illness.

The findings are less applicable to people of African-Caribbean origin who were underrepresented in the sample. Studies in the UK and the US have shown that psychotic disorders in this ethnic group are often misdiagnosed for many reasons. ${ }^{2}$

Diagnostic concordance between the 6 and 24 month evaluations was considerably greater for schizophrenia $(92 \%)$ than for other SSDs such as schizoaffective disorder $(36 \%)$ and schizophreniform disorder $(55 \%)$. Concordance for the diagnosis at 24 months compared with baseline diagnosis, however, was lower for schizophrenia (55\%) than for schizophreniform disorders (86\%), BPD (73\%), and MDD (74\%). The DSM-IV duration criterion of 6 months to establish a diagnosis of schizophrenia provides greater predictive value at the expense of sensitivity determined at initial hospital admission. The World Health Organisation's $I C D-10$ diagnostic guidelines require only 1 month of active symptoms to diagnose schizophrenia and are less restrictive in first admission psychosis with marginal loss of predictive validity. ${ }^{1}$ Clinicians are well advised to review diagnoses periodically taking into account all available information to confirm initial diagnoses irrespective of which diagnostic guidelines are used.

Prathap Tharyan, MD, MRCPsych Christian Medical College Vellore, Tamil Nadu, India

1 Mason P, Harrison G, Croudace T, et al. The predictive validity of a diagnosis of schizophrenia. A report from the International Study of Schizophrenia (ISoS) coordinated by the World Health Organization and the Department of Psychiatry, University of Nottingham. BrJ Psychiatry 1997;1 170:321-7.

2 McKenzie K. Moving the misdiagnosis debate forward. Int Rev Psychiatry 1999;11:153-61. 